\title{
Zur Modellierung der chemischen Absorption im Strahlwäscher
}

\author{
Timo Loos, Josef Schmadl
}

\section{Problemstellung}

In Zeiten globaler Erwärmung und stetig steigender Umweltverschmutzungen spielt der Umweltschutz eine immer stärkere Rolle. Im Mittelpunkt der gesellschaftlichen Diskussion stehen dabei besonders Rauchgase, die bei der Verbrennung fossiler Energieträger zur Energieerzeugung oder in Müllverbrennungsanlagen entstehen. Diese sind mit Flugasche, Staub und Schwermetallen und Sauergasen belastet. Vor allem die Sauergase wie Schwefeldioxid, Schwefelwasserstoff, Chlorwasserstoff und Kohlendioxid sind als kritisch zu betrachten. Das industriell am häufigsten angewandte Verfahren für die Sauergasabtrennung ist die chemische Absorption. Als Waschmittel werden basisch wirkende wässrige Lösungen eingesetzt.

Für eine hohe Effektivität des Absorbers ist ein intensiver Kontakt zwischen Gasgemisch und Waschmittel nötig. Der Strahlwäscher als Gas/Flüssigkeits-Kontaktapparat nimmt dabei eine Sonderstellung ein. Im Gegensatz zum häufig untersuchten Sprühwäscher kann er durch den hohen Energieeintrag des Flüssigkeitsstrahles das Gas ansaugen bzw. verdichten.

Die Stoffaustauschfläche im Strahlwäscher ergibt sich durch die Tropfen und den sich ausbildenden Wandfilm. Der Strahlwäscher ist daher ganz wesentlich durch seine Fluiddynamik gekennzeichnet. Für die Auslegung von Strahlwäschern wurde bisher auf Erfahrungswerte und einfache Modellansätze zurückgegriffen. Diese können die Fluiddynamik nur näherungsweise und für eingeschränkte Betriebsbereiche beschreiben. In diesem Beitrag wird ein kurzer Überblick über Ansätze aus der Literatur gegeben, sowie ein neues rigoroses Modell zur Berechnung eines Strahlwäschers auf Basis einer numerischen Strömungssimulation vorgestellt.

\section{Grundlagen zur chemischen Absorption im Stahlwäscher}

Man unterscheidet zwischen der physikalischen Absorption - hier ist die Gaslöslichkeit durch physikalische Kräfte (van der Waalsche Kräfte) bedingt - und der chemischen Absorption, bei der das Gas eine chemische Bindung mit der Waschflüssigkeit eingeht. In der industriellen Praxis existieren weit mehr chemische als physikalische Absorptionsanlagen.

Für eine hohe Effektivität des Absorbers ist ein intensiver Kontakt zwischen Gasgemisch und Waschmittel nötig. Für die apparative Gestaltung bietet sich die ganze Vielfalt von Gas/Flüssigkeits-Kontaktapparaten an. Absorber, die ihre Austauschfläche nur durch Tropfen infolge der Flüssigkeitszerstäubung erzeugen, haben gegenüber Absorptionskolonnen eine geringere Stoffaustauschfläche. Zu diesen Wäschertypen gehören der Strahl-, der Sprüh- und der Venturiwäscher. Sie werden bevorzugt als chemische Absorber eingesetzt, wenn leicht lösliche Gase bei kurzer Verweilzeit von der Waschflüssigkeit aufgenommen werden sollen.

Im Strahlwäscher strömen Gas und Flüssigkeit im Gleichstrom durch den meist senkrecht angeordneten Wäscher, s. Bild 1. Die Flüssigkeit wird am Kopf versprüht und zerfällt in einen Tropfenschwarm, der als Treibstrahl genutzt wird. Infolge der Differenzgeschwindigkeit zwischen Flüssigkeit und Gas kann durch die Schleppwirkung das Gas angesaugt werden (Strahlpumpenwirkung). Strahlwäscher erfordern einen großen Aufwand an Förderenergie, haben aber einen intensiven Phasenkontakt, ein kleines Bauvolumen und ihr Absorptionsgrad ist weitgehend unabhängig von Schwankungen im Gasstrom. Der Strahlwäscher eignet sich auch zur Staub- und Aerosolabscheidung von groben Partikeln. Gleichzeitig kann durch den Quecheffekt das Abgas gekühlt werden.

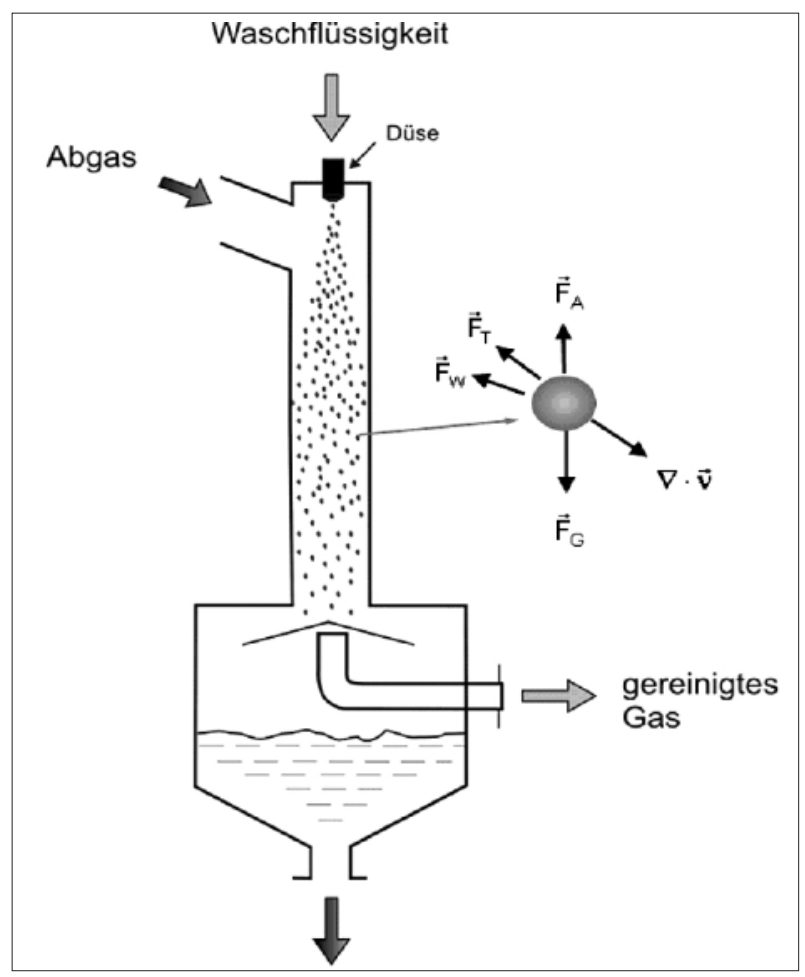

Bild 1: Prinzipskizze des Strahlwäschers

\section{Modellierungsansätze}

\subsection{Empirischer Modelle aus der Literatur}

Ziel der Modellierung eines Strahlwäschers ist die Beschreibung der Hydrodynamik und damit der erreichbaren Pressung und die Berechnung der chemischen Absorption. 
Umfassende Untersuchungen zur Modellierung von Strahlwäschern wurden bereits in den 70er Jahren durchgeführt [5], [2]. Nach Hoffmann [2] kann der Druckverlauf in einem Strahlwäscher nach Gl. (3.1) beschrieben werden.

$\Delta p=\xi \cdot \frac{\rho_{\mathrm{G}}}{2} \cdot \omega_{0}^{2} \cdot \frac{L}{d_{\text {stra }}}$

mit $\mathrm{L} / \mathrm{d}_{\text {stra }}$ dem Geometrieverhältnis und $\xi$ dem Widerstandsbeiwert

$\xi=C_{1} \operatorname{Re}^{-\mathrm{m}} \theta^{-\mathrm{n}}$

mit $\theta$ dem Volumenanteil der Flüssigkeit im Strahlwäscher

Die Konstanten C1, $\mathrm{m}$ und $\mathrm{n}$ müssen über eine Regressionsanalyse bestimmt werden. Der Ansatz gilt nur für hohe Gasgeschwindigkeiten, also einem Strömungsregime in dem die erzeugte Pressung vernachlässigbar gegenüber dem Druckverlust ist.

Im Strahlwäscher ist eine Abhängigkeit der spezifischen Austauschfläche von der dissipierten Energie zu erwarten. Darüber hinaus steigt die Austauschfläche mit dem Flüssigkeitsinhalt an. Hoffmann [2] hat für die Berechnung der spezifischen Austauschfläche in Strahlwäschern einen empirischen Ansatz entwickelt, s. Gl (3.3).

$a_{\text {cff }}=C \cdot\left(\frac{\dot{V}_{1}}{\dot{V}_{1}+\dot{V}_{\mathrm{g}}}\right)^{q} \sqrt[5]{\left(\frac{P}{V_{s t r}}\right)^{2} \frac{\rho_{1}}{\sigma^{3}}}$

mit $\mathrm{P} / \mathrm{V}_{\text {str }}$ der eingetragenen Leistung pro Volumen und den empirischen Konstanten C und q. Über die Konstanten gibt es bisher nur wenige Angaben [4]. In dem Ansatz werden wichtige physikalische Randbedingungen wie die Strahlwäschergeometrie nicht berücksichtigt.

\subsection{Eindimensionales Modell}

Im Gegensatz zu den Füllkörper- und Bodenkolonnen ergibt sich die Stoffaustauschfläche im Strahlwäscher nicht über die einfach zu beschreibende Geometrie der Einbauten, sondern ausschließlich aus der Oberfläche der Tropfen und dem sich ausbildenden Wandfilm. Die konsistente Beschreibung der dispersen Phase in der Zweiphasenströmung scheint daher erforderlich.

Die Sauergaswäsche mittels chemischer Absorption erfolgt für die meisten Anwendungen in einer Kolonne. Die Modellierung der thermodynamisch-mathematischen Zusammenhänge einer Kolonne erfolgt üblicherweise durch eine Kaskade von diskreten Segmenten. Dieser Simulationsansatz ist prinzipiell auch für Strahlwäscher möglich. Die Bilanz und Transportgleichungen ergeben ein differentiell-algebraisches Gleichungssystem, das zum Beispiel mit dem Runge-Kutta-Verfahren numerisch gelöst werden kann.

Zur Berechnung der Pressung muss die Geschwindigkeit der Tropfen bekannt sein. Die Impulsänderung eines Tropfens erhält man über das Kräftegleichgewicht am Tropfen, s. Bild 1. Die Kräfte, die auf den Tropfen wirken, sind die Gewichtskraft $\vec{F}_{\mathrm{G}}$, die Auftriebskraft $\vec{F}_{\mathrm{A}}$, die Widerstandskraft $\vec{F}_{\mathrm{W}}$ und die Trägheitskraft $\vec{F}_{\mathrm{T}}$.

Die Trägheitskraft ergibt sich aus der Impulsänderung eines Tropfens. Vernachlässigt man die Änderung der Masse, gilt für die Impulsänderung des Tropfens Gl. (3.4). $m \frac{\mathrm{d} \vec{v}}{\mathrm{~d} t}=\sum_{i} \vec{F}_{\mathrm{i}}$

Für die Kräftebilanz werden die Vektoren in vertikale und horizontale Komponenten zerlegt. Für die vertikale Kräftebilanz (z-Koordinate) müssen die Auftriebs- und Gewichtskraft berücksichtigt werden.

$\sum F_{\mathrm{z}}=F_{\mathrm{G}, \mathrm{z}}-F_{\mathrm{T}, \mathrm{z}}-F_{\mathrm{A}, \mathrm{z}}-F_{\mathrm{W}, \mathrm{z}}=0$

Unter Annahme einer vertikalen Kolbenströmung des Gases folgt aus der vertikalen Kräftebilanz

$\frac{\mathrm{d} v_{\mathrm{p}, L}}{\mathrm{~d} t}=g \cdot\left(1-\frac{\rho_{\mathrm{g}}}{\rho_{1}}\right)-c_{w} \cdot \frac{3}{4} \cdot \frac{\rho_{\mathrm{g}}}{\rho_{1}} \cdot \frac{1}{d_{\mathrm{p}}}\left(v_{\mathrm{p}, \mathrm{z}}-v_{\mathrm{g}, \mathrm{z}}\right) \cdot \mid v_{\mathrm{p}, \mathrm{z}}-v_{\mathrm{g}, \mathrm{z}}$

Für die Wechselwirkung zwischen der Gasphase und der dispersen Phase ist der Widerstandsbeiwert $\left(\mathrm{c}_{\mathrm{w}}\right)$ maßgebliche Einflussgröße, der das aerodynamische Widerstandsverhalten eines Tropfens mit der umgebenden Phase beschreibt. Für die Berechnung von Widerstandsbeiwerten von Tropfen geht man meistens von einer starren Kugelform aus. Die Korrelationen der Widerstandsbeiwerte werden in Abhängigkeit von der Partikelreynoldszahl beschrieben, s. Gl. (3.7).

$R e_{\mathrm{p}}=\frac{v_{\mathrm{rel}} \cdot d_{\mathrm{p}}}{v}$

Der Widerstandsbeiwert für eine laminare, schleichende Umströmung einer Kugel wird mit dem Stokes'schen Gesetz beschrieben. Unter turbulenten Strömmungsbedingungen hat das Stokes'sche Gesetz keine Gültigkeit mehr. Der Ansatz nach Brauer [1] gilt bis zur kritischen $R e_{\mathrm{p}}=3 \cdot 10^{5}$ :

$c_{w}=\frac{24}{R e_{p}}+\frac{3,73}{R e_{\mathrm{p}}^{0,5}}-\frac{4,83 \cdot 10^{-3} \cdot R e_{\mathrm{p}}^{0,5}}{1+3 \cdot 10^{-6} \cdot R e_{\mathrm{p}}^{1,5}}+0,49$

Die Korrelationen basieren auf Messungen an Einzelkugeln und -Tropfen. Zumindest im düsennahen Bereich kommt es zu einer Interaktion der Tropfen. Für diesen Effekt existiert noch keine gesicherte Korrelation, so dass die Interaktion im düsennahen Bereich vernachlässigt werden muss.

Die Pressung im Strahlwäscher bzw. der Unterdruck im Ansaugrohr entsteht durch die beschleunigten Flüssigkeitstropfen. Für eine stationäre Gasströmung kann die Pressung nach Gl. (3.9) berechnet werden. Der erste Term der rechten Seite beschreibt den Druckverlust der Rohrströmung, der Zweite die erzeugte Pressung durch die Tropfen.

$\frac{\partial p}{\partial z}=-\frac{1}{2} \cdot \frac{\lambda}{D} \cdot \rho_{g} \cdot v_{g}^{2}+\frac{1}{8} \pi \cdot c_{w}\left(v_{p, Z}\right) \cdot \varphi(z) \cdot \rho_{g} \cdot d_{p}^{2} \cdot\left(v_{p, z}-v_{g}\right) \cdot \mid v_{p, z}-v_{g}$

mit $\varphi$ der Tropfenanzahl pro Volumen und der Rohreibungszahl $\lambda$

Durch die tatsächliche Berechnung der dispersen Phase über Gl. (3.8) und (3.9) ist das eindimensionale Zellenmodell genauer als die empirischen Ansätze. Es müssen jedoch wesentliche Vereinfachungen getroffen werden: die Phasen sind gleichmäßig über den Querschnitt verteilt, das Gas weist in axialer Richtung eine Kolbenströmung auf, die Geschwindigkeitskomponenten in radialer und 
Umfangsrichtung werden vernachlässig und die Tropfenzahl wird als konstant angenommen. Dadurch ist die Berechnung der Pressung immer noch sehr ungenau. Für die Berechnung der Absorption werden die Fehler mit dem eindimensionalen Modell noch größer.

\section{Rigoroses Modell}

Die numerische Strömungssimulation berechnet das tatsächliche Verhalten der Phasen und gehört zu den exakten Modellen. Heutzutage wird die numerische Simulation in vielen wissenschaftlichen und industriellen Bereichen angewendet. Der wichtigste Anwendungsbereich ist die Simulation von Strömungen. Die Impuls-, Wärme- und Stofftransportvorgänge sowie die geometrischen und fluiddynamischen Randbedingungen werden berücksichtigt.

In den meisten Strahlwäschern erfolgt die Gaszufuhr seitlich. Durch eine zweidimensionale Betrachtung kann diese Strömungsführung nicht exakt berechnet werden. Des Weiteren sind Turbulenzwirbel grundsätzlich dreidimensional. Es empfiehlt sich daher eine dreidimensionale Strömungssimulation

\subsection{Berechnung der Gasphase nach Euler}

Entscheidend für die Berechung von Strömungen sind die Wechselwirkungen zwischen den Fluidpartikeln untereinander und zwischen dem Fluid und einem ruhenden oder bewegten Körpers.

Beim Euler-Modell wird die Individualität der Moleküle nicht berücksichtigt, das heißt das Strömungsmedium wird als Kontinuum betrachtet. Man spricht beim Euler-Modell von einer ortsfesten Beobachtung, da die Bilanzierung für ein ortsfestes Kontrollvolumen erfolgt.

Die Strömung wird durch die Navier-Stokes'schenGleichungen beschrieben. Diese lassen sich aus den Erhaltungsgleichungen für Masse und Impuls herleiten. Die Gleichung für die Massenerhaltung setzt sich aus einem Term, der die zeitliche Änderung der Masse, und einem Term, der die Änderung der Masse durch Konvektion beschreibt, zusammen.

$\frac{\partial \rho}{\partial \mathrm{t}}+\frac{\partial}{\partial x_{\mathrm{i}}}\left(\rho v_{\mathrm{i}}\right)=0$

Die Impulsbilanz lässt sich an einem finiten Volumenelement für das kartesischen Koordinatensystem nach Gl. (4.11) beschreiben [6].

$\frac{\partial}{\partial t}\left(\rho v_{\mathrm{i}}\right)+\frac{\partial}{\partial x_{\mathrm{j}}}\left(\rho v_{\mathrm{i}} v_{\mathrm{j}}\right)=\frac{\partial}{\partial x_{\mathrm{j}}} \tau_{\mathrm{i}, \mathrm{j}}-\frac{\partial p}{\partial x_{\mathrm{j}}}+\rho \cdot g_{\mathrm{i}}+F_{\mathrm{i}}$

mit $\mathrm{i}$ und $\mathrm{j}$ dem Richtungsindex

Die Terme der Gl. (4.11) beschreiben die zeitliche Änderung des Impulses, den Impulstransport durch Konvektion, die Änderungen der Schub - und Normalspannungen sowie den Quelltermen bestehend aus einem Druckterm, der Gravitationskraft und äußeren Kräften $\left(\mathrm{F}_{\mathrm{i}}\right)$.

Das orts- und zeitabhängige System der partiellen Differentialgleichungen lässt sich nur numerisch lösen. Das Berechnungsgebiet wird diskretisiert, das heißt es werden nur endlich viele ausgewählte Volumina betrachtet. Das diskrete Modell muss das kontinuierliche Modell möglichst gut approximieren. Die Erhaltungsgleichungen für Masse, und Impuls werden für jedes einzelne Volumen integriert und gelöst.

\subsection{Berechnung der dispersen Phase}

Die Berechnung der dispersen Phase erfolgt nach der Methode von Lagrange. Die Tropfengeschwindigkeit wird zeitabhängig entlang des Weges berechnet. Das heißt, die Bahnlinien eines Masseteils werden im System verfolgt. Man spricht daher im Gegensatz zum Euler-Modell von einem mitbewegten Beobachter. Die Impulsänderung der dispersen Phase wird, wie für das eindimensionale Modell über das Kräftegleichgewicht am Tropfen berechnet.

Die Tropfeninjektion im Simulationsmodell hat einen wesentlichen Einfluss auf die Verteilung der dispersen Phase. Die Tropfen können im Modell nicht einzeln berechnet werden, so werden mehrere Tropfen zu einem Tropfenschwarm mit gleichen Eigenschaften zusammengefasst. Bei der Anzahl der Tropfenschwärme gilt es ein Optimum zwischen Genauigkeit und Rechenaufwand zu finden.

Aufgrund des hohen Impulses der Tropfen im Strahlwäscher werden die Tropfen kaum abgelenkt. Der Abstand der Tropfen nimmt daher durch die Strahlaufweitung mit der Entfernung von der Düse zu. Die Koaleszenz von Tropfen ist daher als gering einzuschätzen.

\subsection{Turbulenzmodellierung}

In einer turbulenten Strömung bewegen sich die Fluidteilchen völlig ungeordnet um die dominierende Hauptströmung. Der Transport von Impuls und Wärme erfolgt nicht nur entlang der Hauptströmungsrichtung, sondern auch durch die turbulente Querströmung.

Zur Modellierung der Turbulenz haben sich Zweigleichungsmodelle bewährt, zu denen das k-ع-Modell, das kL-Modell und das k- $\omega$-Modell zählen. Das semi-empirische k-ع-Modell ist besonders für hochturbulente Strömungen anwendbar und bietet sich daher für die Modellierung der Turbulenz in einem Strahlwäscher an.

\subsection{Berechnung der chemischen Absorption}

Die meisten kinetischen Ansätze für reaktive Trennprozesse werden durch das von Lewis und Whitman [3] aufgestellte Zwei-Film-Modell beschrieben. Das Modell basiert auf dem Fickschen Gesetz. Die an den Filmen angrenzenden Kernphasen werden als ideal durchmischt betrachtet. An der Phasengrenzfläche wird vollständiges thermodynamisches Gleichgewicht vorausgesetzt.

Der Stofftransport der zu absorbierenden Komponente erfolgt aus der Gasphase in die Flüssigphase. Die Beschreibung der Löslichkeit einer Gaskomponente in einer Flüssigkeit kann mit dem Henryschen Gesetz erfolgen. Geht der Stoffmengenanteil in einer ideal verdünnten Lösung gegen Null $\left(\mathrm{x}_{\mathrm{i}} \rightarrow 0\right)$ kann das eingestellte Phasengleichgewicht nach Gl. (4.12) beschrieben werden.

$p_{\mathrm{i}}=H \cdot x_{\mathrm{i}}$

Unter Berücksichtigung der chemischen Absorption kann der Stoffübergang vereinfacht beschrieben werden [7]:

- Diffusion der absorbierbaren Komponente durch die Gasphase zur Phasengrenzfläche 
- Übergang der Komponente in die Flüssigkeit

- Diffusion der Komponente und deren Reaktionspartner in der Flüssigkeitsphase zur

- Reaktionszone

- Ablauf der chemischen Reaktion

- Transport der Reaktionsprodukte in den Kern der Flüssigkeit

Für geringe Gaskonzentrationen kann man von linearen Konzentrationsprofilen in den Grenzschichten ausgehen, s. Bild 2.

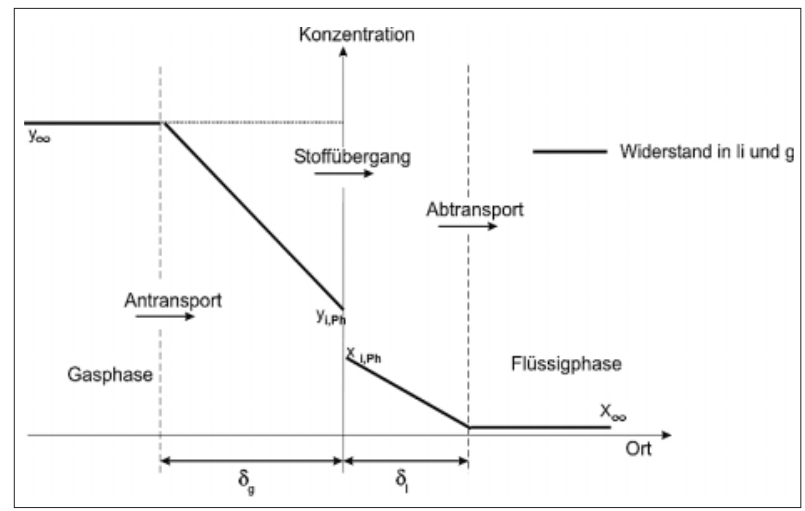

Bild 2: Zweifilmtheorie

Der Stoffstrom für beide Phasen durch die Phasengrenzfläche A kann mit Hilfe des flüssigkeitsseitigen Stoffübergangskoeffizienten $\beta_{f}$ oder dem gasseitigen Stoffübergangskoeffizienten $\beta_{g}$ beschrieben werden.

$\dot{N}_{\mathrm{i}}=\beta_{\mathrm{g}} \cdot A_{\mathrm{Ph}} \cdot c_{\mathrm{tol}, \mathrm{g}} \cdot\left(y_{l, \infty}-y_{\mathrm{i}, \mathrm{Ph}}\right)=\beta_{1} \cdot A_{\mathrm{Ph}} \cdot c_{\mathrm{to} t, \mathrm{l}} \cdot\left(x_{\mathrm{i}, \mathrm{Ph}}-x_{\mathrm{i}, \infty_{\mathrm{o}}}\right)$

Ein Stofftransport mit gleichzeitiger chemischer Reaktion der übergehenden Komponente erhöht die Aufnahmekapazität und den flüssigseitigen Stoffübergangskoeffizienten im Falle einer schnellen Reaktion in der flüssigen Phase. Für die Modellierung des Stofftransportes einer Absorption mit chemischer Reaktion muss die Kinetik der Reaktion zusätzlich zum physikalischen Modell berücksichtigt werden.

Eine weit verbreitete Möglichkeit zur Beschreibung des Einflusses des Stofftransportes durch eine chemische Reaktion ist das Konzept der Enhancementfaktoren. Dieser beschreibt die Erhöhung des Stoffübergangskoeffizienten infolge der chemischen Reaktion gegenüber der reinen physikalischen Absorption.

$E=\frac{\beta_{1, \mathrm{r}}}{\beta_{1}}$

Der gasseitige Stoffübergangskoeffizient beschreibt den Stoffübergang zwischen der Gasseite und der Gas/FlüssigPhasengrenze. Er lässt sich als Funktion der Sherwood-Zahl beschreiben.

$\beta_{\mathrm{g}}=S h \frac{D_{\mathrm{g}}}{d_{\mathrm{p}}}$

Die Bestimmung der Sherwood-Zahl erfolgt meist aus empirisch ermittelten Kennzahl-Korrelationen, basierend auf der Formulierung des Zusammenhangs zwischen der Sherwood-Zahl (Sh), der Reynolds-Zahl (Re) und der Schmidt-Zahl (Sc).

\subsection{Berechnungsablauf}

Für die Berechnung der chemischen Absorption bieten numerische Strömungssimulationsprogramme zurzeit keine Modelle, die diese ausreichend beschreiben. Es muss daher ein eigenes Modell entwickelt und in die Strömungssimulation implementiert werden [8]. Das Modell kann in drei Prozeduren unterteilt werden.

In der ersten Prozedur wird der Stoffübergang berechnet. Trifft ein Tropfenstrom in eine neue Zelle, wird dessen repräsentierende Gesamtoberfläche und die Verweilzeit in der Zelle erfasst, s. Bild 3. Der übergehende Stoffstrom wird nach dem oben beschriebenen Zweifilmmodell berechnet.

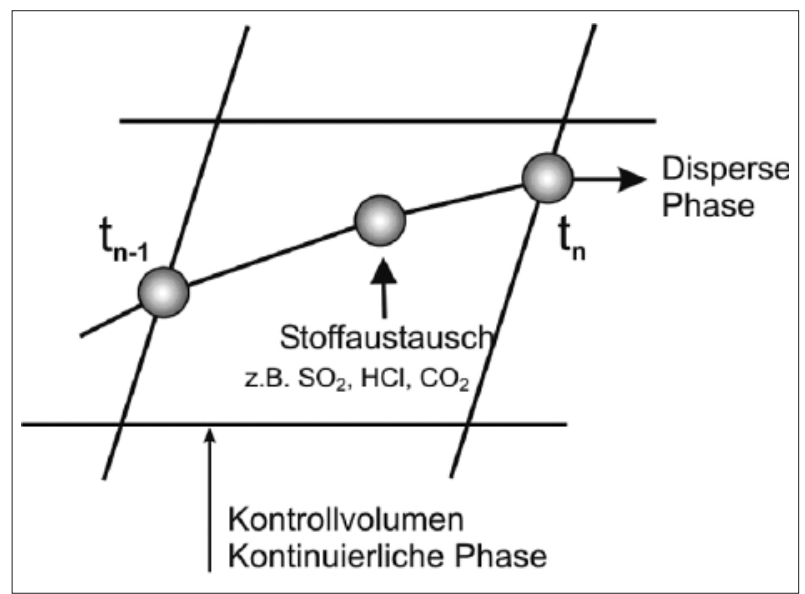

Bild 3: Prinzip Stoffaustausch in einem Kontrollvolumen

Eine direkte Simulation des Wandfilmes ist aufgrund der hohen Rechenzeiten nicht praktikabel. Da der Wandfilm die Hydrodynamik nur unwesentlich beeinflusst, wird er nur fiktiv zur Berechnung der Absorption berücksichtigt.

Tropfen, die auf die Wand treffen, werden in einer zweiten Prozedur aufsummiert, so dass die Reynolds-Zahl des Filmes berechnet werden kann. Für den Wandfilmbereich lässt sich so ebenfalls ein übergehender Stoffstrom berechnen.

In der dritten Prozedur wird der übergegangene Stoffstrom als Senke an die kontinuierliche Phase zurückgegeben. Die Iteration wird so lange fortgesetzt, bis das vorgeschriebene Konvergenzkriterium erreicht wird, s. Bild 4.

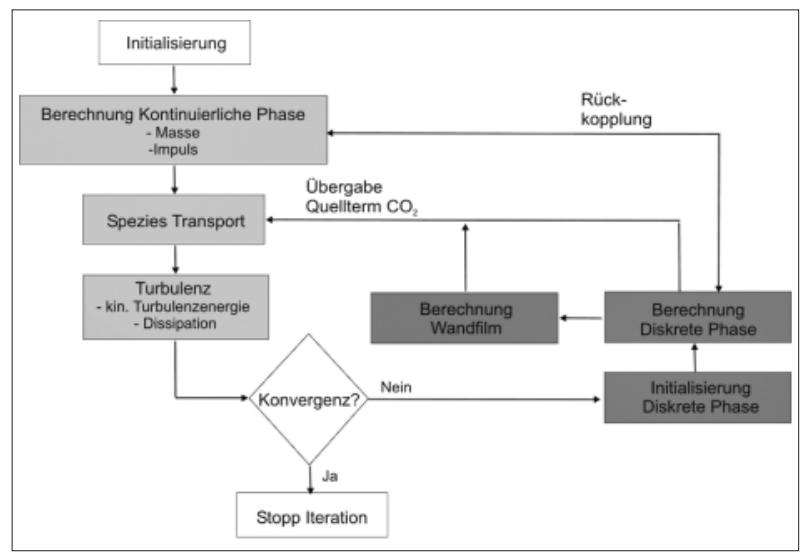

Bild 4: Berechnungsschema rigoroses Modell

Mit einer dreidimensionalen Strömungssimulation können die tatsächlichen Strömungsbedingungen im Strahlwäscher ohne direkte Anpassung der Ergebnisse berechnet 
werden. Durch die Kenntnis des Strömungsregimes können die Verweilzeiten, die lokale Relativgeschwindigkeit und damit der lokale Stoffaustausch berechnet werden. Dadurch kann der Absorptionsgrad für jeden Bereich im Strahlwäscher bestimmt werden. Die Implementierung der Absorption über eine eigene Berechnungsprozedur erlaubt eine Erweiterung für beliebige Stoffsysteme. Das Modell ist auch auf den häufig verwendeten, jedoch aus strömungstechnischer Sicht weniger anspruchsvollen Sprühwäscher übertragbar.

\section{Literatur}

[1] Brauer, H.; Impuls-, Stoff- und Wärmetransport durch die Grenzfläche kugelförmiger Partikel; Chem. Ing. Tech. 45 (1973) 18, 1099-1103.

[2] Hoffmann, R., Kürten, H., Nagel, O.; Stoffaustauschfläche und Hydrodynamik in Strahl- bzw. Venturi-Wäschern; Chem. Ing. Tech. 45 (1973) 13; 881-887.

[3] Lewis, W. K., Whitman, W. G.; Principles of Gas Absorption; Ind. Eng. Chem. 19 (1924), 1215-1220.

[4] Mersmann, A., Hofer, H., Stichlmair, J.; Absorption und Absorber; Chem. Ing. Tech. 51 (1979) 3; 157-166.

[5] Nagel, O., Kürten, H., Hegner, B.; Die Stoffaustauschfläche in Gas/Flüssigkeits-Kontaktapparaten Auswahlkriterien und Unterlagen zur Vergrößerung; Chem. Ing. Tech. 45 (1973) 14; 913-920.

[6] Schacht, W.; Verfahren für die Berechnung von Kennlinien von Flüssigstrahl-Ventilatoren und Strahlwäscher-Anlagen; Chem. Ing. Tech. 64 (1992) 4; 382-383.

[7] Schmadl, J.; Vorlesungsskript Wärme- und Stoffübertragung; TFH-Wildau

[8] Loss, T., Dissertation Universität Dresden, in Vorbereitung.

\section{Autoren}

Dipl.-Ing. (FH) Timo Loos

Technische Fachhochschule Wildau

Fachbereich Ingenieurwesen/Wirtschaftsingenieurwesen

Prof. Dr.-Ing. Josef Schmadl

Technische Fachhochschule Wildau

Fachbereich Ingenieurwesen/Wirtschaftsingenieurwesen Tel. +49 3375 508-110

jschmadl@igw.tfh-wildau.de 